\title{
ORDENACIÓN SANITARIA EN EL MEDIO RURAL DE LA PROVINCIA DE ALICANTE*
}

\author{
J. Fermín Bonmatí Antón
}

\section{RESUMEN}

En el plano sanitario, la población alicantina residente en los espacios más ruralizados se encuentra en desventaja respecto a los demás habitantes de la provincia. Por un lado, suele coincidir este censo con el de los pacientes con menor accesibilidad a los respectivos centros hospitalarios, hecho que se subsana con la presencia de Consultorios de Asistencia Primaria y Centros de Salud más próximos a las áreas rurales. Por otra parte, se trata de un contingente muy envejecido, con porcentajes muy elevados de población con 60 o más años de edad, circunstancia a tener en cuenta en la planificación y ordenación territorial de los recursos sanitarios.

\section{RESUME}

Du point de vue de la santé la population alicantinéenne qui vit aux espaces plus ruraux se trouve avec désavantage sur la reste de la province. Elle coïncide avec le groupe de patients plus éloignés des hôpitals; cependant il y a de cabinets de consultation et de Centres de la Santé aux villes proches. En autre, il s'agil d'une population très agée, avec pourcentages importants de personnes avec 60 ou plus années, qu'il faut n'en oublier pas pour la planification et l'arrangement des recours des soins.

\section{La Geografía de la Salud: una disciplina poco estudiada}

La Geografía de la Salud o Geografía Médica es una rama poco conocida y apenas practicada en España, aunque existen precedentes en estudios geográficos y topografías médicas durante el siglo XIX, sobre todo en Francia, y mucho más en los países anglosajones. En Francia, cabe reseñar, como excepcional e importante aportación, los estudios de

* Esta investigación se ha realizado dentro del Convenio Universidad de Alicante-Diputación Provincial de Alicante, B.6 / 90, titulado «Bases demográficas, recursos económicos y dotaciones serviciales en los municipios rurales de la provincia de Alicante». 
Max Sorre entre los años veinte y sesenta del presente siglo ${ }^{1}$, y de Henri Pichéral ${ }^{2}$ y J. P. Besancenot $^{3}$ a partir de los años setenta.

Sin embargo, lo esencial se encuentra en monografías y revistas de Medicina o Geografía anglosajonas, destacando la fundamental recopilación de N. D. McGlashan ${ }^{4}$ o los estudios de J. M. Mey en los años cincuenta ${ }^{5}$ y de A. T. A. Learmonth ${ }^{6}$.

Desde 1970 la disciplina — denominada Geografía de la Salud a partir del Congreso de la U.G.I. celebrado en Moscú, en 1976-, experimenta un despegue, sobresaliendo autores como P. Hagget, A. D. Cliff, G. M. Howe (R. Unido), J. P. Thouez, A. E. Joseph (Canadá), G. F. Pyle, M. Meade (EE.UU.). En los últimos Congresos de la U.G.I., celebrados en Japón (1980), Francia (1984), Australia (1988) y EE.UU. (1992), se ha puesto de manifiesto la preeminencia de los geógrafos norteamericanos en el seno de las respectivas Comisiones de Geografía de la Salud, dada la abrumadora mayoría de comunicaciones presentadas por dichos autores.

Cabe aplaudir, por ello, iniciativas como las de la revista francesa Espace, Populations, Sociétés, cuyo número 3 del año 1992 corresponde a un monográfico dedicado a la salud. En este ejemplar, algunos de los mejores especialistas (H. Pichéral, F. Tonnellier, E. Vigneron, M. Flint, N. McGlashan) tratan de dar respuesta a la cuestión sobre si la igualdad y la justicia social están comprometidas por la desequilibrada distribución de los recursos sanitarios en el espacio, a través del estudio de la situación en distintos puntos del planeta (EE.UU., Francia, Polinesia, Tasmania).

En España, la Geografía de la Salud es una gran desconocida y sus relaciones con la demografía aún más, aunque en los últimos años se han publicado algunos estudios. Así, contamos con el libro de síntesis de A. Olivera ${ }^{7}$, y los trabajos de E. Aracil ${ }^{8}$, de B. González ${ }^{9}$, de A. Cañis ${ }^{10}$, de J. Carrasco y J. Fereres ${ }^{11}$, de J. M. Comelles y A. Martínez-Hernáez ${ }^{12}$, de M. A. Durán ${ }^{13}$, de P. Jaén ${ }^{14}$, de M. A. López de los Mozos y C.

1 SORRE, M.: «Complexes pathogènes et Géographie médicale», Annales de Géographie, 1933. Véase también, del mismo autor: Les fondement biologiques de la Géographie humaine. Essai d'une écologie de l'homme, A. Colin, Paris, 1943; y «Géographie de l'état sanitaire et des maladies», en Géographie Générale. Encyclopedie de la Pléiade, Gallimard, Paris, 1966.

2 Véase, p.ej., su Tesis Doctoral: PICHERAL, H.: Espace et santé. Géographie médicale du Midi de la France, Université, Montpellier, 1976.

3 BESANCENOT, J. P.: «Vents et santé en façade méditerranéenne de l'Europe», Annales de Géographie, núm. 546 (Paris, 1989), pp. 179-195.

4 McGLASHAN, N.D. (dir.): Medical Geography, Methuen, Londres, 1972.

5 P.ej., MEY, J.M.: «Medical Geography, its methods and objectives», Geographical Review, 1951, pp. 941.

6 LEARMONTH, A.T.A.: «Medicine and medical geography», en McGLASHAN, N.D. (dir.): Op. cit.

7 OLIVERA POLL, A.: Geografía de la Salud, Síntesis, Madrid, 1993, 160 pp.

8 ARACIL RODRÍGUEZ, E.: «Tendencias demográficas y planificación de recursos sanitarios», II Jornadas Internacionales sobre Demografía Urbana y Regional, Instituto de Demografía del C.S.I.C. (Valencia, 1992). También, de esta autora, «El análisis demográfico en la planificación de recursos sanitarios», IV Jornadas de la Población Española, Universidad de La Laguna y A.G.E., Santa Cruz de Tenerife, 1993, pp. 427-437.

9 GONZÁLEZ RODRÍGUEZ, B.: El capital humano en el sector sanitario: La distribución de médicos en España, C.S.I.C., Madrid, 1979, 115 pp.

10 CAÑIS PREDENY, A.: «Cambio de la estructura de edad de la población y cambio de la demanda sanitaria», Todo Hospital, núm. 84 (Madrid, 1992), pp. 11-15.

11 CARRASCO ASENJO, J.-FERERES CASTIEL, J.: Estudio del área de influencia del Hospital General de Madrid, Madrid, 1981.

12 COMELLES, J. M. - MARTÍNEZ-HERNAEZ, A.: Enfermedad, cultura y sociedad, EUDEMA, Madrid, 1993, $96 \mathrm{pp}$.

13 DURÁN, M. A.: Desigualdad social y enfermedad, Tecnos, Madrid, 1983, 180 pp.

14 JAÉN LUQUE, P.: «Estructura espacial del equipamiento sanitario en Madrid», Bol. Real Soc. Geográfica, 1987, pp. 131-166. 
Espejo $^{15}$, y, en general, los del Instituto de Demografía del C.S.I.C. Sobre la infraestructura sanitaria en la Comunidad Valenciana se cuenta con los trabajos de J. Costa ${ }^{16}$. Otras aportaciones interesantes inciden en la planificación sanitaria con miras a satisfacer las demandas de sectores específicos de la población. Así, en relación con la población más envejecida, cabe citar los estudios de Guerrero et al. ${ }^{17}$, de G. Fernández-Mayoralas para un área de salud en la provincia de Badajoz ${ }^{18}$, de A. Rodríguez y A. Abellán sobre el Hospital madrileño «Puerta de Hierro» ${ }^{19}$, de J. M. González y R. Rodríguez sobre el espacio rural gallego ${ }^{20}$ y de V. Rodríguez para Castilla-La Mancha ${ }^{21}$.

\section{Fuentes y metodología}

En el presente trabajo, la atención se centra en las estructuras, dotaciones y servicios sanitarios del espacio rural en la provincia de Alicante, haciendo especial hincapié en los desequilibrios municipales en materia de dotaciones y en la tendencia a la concentración urbana de las mismas. Por esta razón, no se analizarán exclusivamente los datos relativos a las entidades municipales con menor censo de la provincia (con menos de 2.000 habitantes de hecho en cabecera en 1991 — según el listado que figura en el mapa 1-), sino también los de localidades con mayor rango demográfico, distinguiendo — según conveniencia o no- entre censo en cabecera y en el resto del territorio municipal, con objeto de calibrar la importancia relativa de los equipamientos y servicios que se dan en aquéllos dentro de su correspondiente contexto comarcal.

Se aplica la división geográfica establecida por el Decreto 46/1986 y la Ley 8/1987 de 4 de diciembre de 1987 en Áreas de Salud (véase mapas 1 y 2), definidas como la demarcación espacial y poblacional en que se lleva a cabo la planificación, programación, gestión y coordinación de la atención a la salud, y que abarca, en principio, poblaciones entre doscientos y doscientos cincuenta mil habitantes. Las Areas constituyen la estructura fundamental en la ordenación del moderno sistema sanitario del Servei Valencià de Salut (SERVASA), organismo público de la Generalitat que asumió todas las competencias en materia de sanidad en la Comunidad Valenciana a partir de 1988 en virtud del R.D. 1.612/ 87 de 27 de diciembre de 1987. A su vez, las Áreas coinciden con los denominados Distritos Sanitarios, que se ordenan a partir de ocho Hospitales Generales, salvo la de

15 LÓPEZ DE LOS MOZOS, M.A. - ESPEJO, C.: «El equipamiento hospitalario en la ciudad de Madrid», Estudios Territoriales, 37 (Madrid, 1991), pp. 107-119.

16 COSTA MAS, J.: «Los recursos sanitarios en las tierras valencianas», art. inédito, $11 \mathrm{ff}$. También, en capítulo compartido, COSTA MAS, J. - BARTOLOMÉ PINA P.A.: «Recursos sanitarios y educativos», en AA.VV.: Atlas Temático de la Comunidad Valenciana, Diario Información, Alicante, 1991, pp. 681-700.

17 GUERRERO, M. - ALFONSO, J.L. - SANCHIS, B. - PRADO, M.J.: «Demanda hospitalaria geriátrica de la población», Actas de las XI Jornadas de Economía de la Salud, Generalitat Valenciana y Asociación de Economía de la Salud (Valencia, 1992), pp. 235-243.

18 FERNÁNDEZ-MAYORALAS FERNÁNDEZ, G.: «Accesibilidad espacio-temporal a los servicios sanitarios hospitalarios de la población de más de sesenta años en un área de salud rural», II Jornadas de la Población Española, Universidad de las Islas Baleares y A.G.E., Palma de Mallorca, 1989, pp. 185-195.

19 RODRÍGUEZ POSE, A.-ABELLÁN GARCÍA, A.: «Estado médico y problemas sociales de personas de edad en régimen hospitalario: el caso del Hospital Puerta de Hierro (Madrid)», II Jornadas de la Población Española, Universidad de las Islas Baleares y A.G.E., Palma de Mallorca, 1989, pp. 371-380.

20 GONZÁLEZ PÉREZ, J.M.-RODRÍGUEZ GONZÁLEZ, R.: «Incidencia del envejecimiento en Galicia y ordenación sanitaria de las áreas rurales», IV Jornadas de la Población Española, Universidad de La Laguna y A.G.E., Santa Cruz de Tenerife, 1993, pp. 343-350.

21 RODRÍGUEZ, V. et al.: Envejecimiento de la población y servicios sociales en Castilla - La Mancha, C.S.I.C. / Instituto de Economía y Geografía Aplicadas, Doc. de Trabajo núm. 9, Madrid, 1990, 62 pp. 
FIGURA 1. Relación de municipios con menos de 2.000 habitantes de hecho en cabecera (en 1991), en la provincia de Alicante, por Distritos Sanitarios:

Distrito XVII (DENIA): 25) V. Gallinera; 26) Adsubia; 27) V. Ebo; 28) V. Alcalá; 30) V. Laguart; 31) Benichembla; 32) Parcent; 33) Murla; 34) Orba; 35) Tormos; 36) Sagra; 37) Ràfol de Almunia; 38) Benimeli; 39) Sanet y Negrals; 40) Beniarbeig; 41) Benidoleig; 42) Alcalalí; 43) Jalón; 44) Llíber; 45) Senija; 46; Benitachell; 47) Setla-Mirarrosa-Miraflor.

Distrito XVIII (VILLAJOYOSA): 48) Tárbena; 49) Bolulla; 50) Guadalest; 51) Polop; 52) Finestrat; 53) Benimantell; 54) Benifato; 55) Beniardá; 56) Confrides; 57) Sella; 58) Orcheta; 59) Relleu. Distrito XIX (ALCOY):1) Alfafara; 2) Agres; 3) Gayanes; 4) Alcocer de Planes; 5) Benimarfull; 6) Alq. Aznar; 7) Benillup; 8) Millena; 9) Gorga; 10) Benilloba; 11) Penáguila; 12) Benifallim; 13) Alcolecha; 14) Benasau; 15) Cuatretondeta; 16) Facheca; 17) Famorca; 18) Tollos; 19) Benimassot; 20) Balones; 21) Almudaina; 22) Planes; 23) Beniarrés; 24) Lorcha; 29) C. Castells.

Distrito XX (ELDA): 64) Benejama; 65) C. Mirra; 66) Cañada; 67) Salinas; 68) Algueña; 69) La Romana; 70) H. Nieves; 71) H. Frailes.

Distrito XXI (SAN JUAN): 60) Torremanzanas; 61) Busot; 62) Aigües de Busot; 63) Tibi.

Distrito XXII (ALICANTE):

Distrito XXIII (ELCHE):

Distrito XXIV (ORIHUELA): 72) Benferri; 73) Gr. Rocamora; 74) S. Fulgencio; 75) Daya Vja.; 76) Daya Nva.; 77) Formentera; 78) Benijófar; 79) Algorfa; 80) Jacarilla. 
l'Alacantí, subdividida en dos Distritos a partir de los Hospitales de Alicante y de San Juan.

Los municipios rurales (con menos de 2.000 habitantes), se integran en los respectivos Distritos de cobertura de las distintas instalaciones hospitalarias, sin perjuicio de que, para determinadas asistencias muy específicas, los pacientes deban trasladarse a los Hospitales que dispongan de los equipos necesarios (Alicante, Elche, Valencia) por carecer de ellos los centros que encabezan los Distritos de salud correspondientes.

En la elaboración del presente estudio se han utilizado cifras estadísticas publicadas por organismos oficiales (Institut Valencià d'Estadística, Consellería d'Economía i Hisenda, Consellería de Sanitat i Consum, Instituto Nacional de Estadística) y también privados (PREVASA - Gabinete de Estudios de la antigua Caja de Ahorros de Valencia, hoy Bancaja-, Caja de Ahorros del Mediterráneo); además, se ha procurado actualizar en lo posibletoda la información relativa a los niveles de equipamientos y servicios en municipios con menos de 2.000 habitantes en la provincia de Alicante, mediante la realización de encuestas personales a los funcionarios de los respectivos Ayuntamientos entre octubre de 1992 y mayo de 1993.

\section{Asistencia Hospitalaria: distribución geográfica e isocronas de acceso a los Hospi- tales de la provincia de Alicante}

Los municipios del Corredor del Vinalopó pertenecen al Distrito sanitario que centraliza el Hospital de Elda, a la expectativa de que los del Alto Vinalopó se integren en el futuro en un área específica capitalizada por las instalaciones hospitalarias que se vienen reclamando para Villena. Los del Bajo Segura pertenecen al Hospital Comarcal de San Bartolomé (pedanía rural del término municipal de Orihuela), el de más reciente inauguración (en 1990). Los de l'Alacantí se sirven de las instalaciones hospitalarias de Alicante y de San Juan. En la Marina Baixa, los servicios asistenciales los jerarquiza el Hospital de Villajoyosa. En la Marina Alta, el centro hospitalario de La Pedrera (Denia). Y en la Montaña, el Hospital «Virgen de los Lirios», ubicado en la ciudad de Alcoy.

Las clínicas de carácter privado se concentran en las poblaciones más desarrolladas de cada comarca y con áreas de influencia sanitaria propia muy difícil de determinar.

También cabe recordar la presencia del único centro hospitalario existente en la actualidad en un municipio «rural» de la provincia de Alicante (tras el cierre, en los años sesenta, de las instalaciones de Aigües de Busot y de Torremanzanas): el Sanatorio de Fontilles, en Vall de Laguart (Marina Alta). Este fue fundado en 1909, para la atención exclusiva a enfermos de lepra. Dispone de 240 camas —en 1989—. Se trata de un centro privado de carácter benéfico, que subsiste mediante donativos particulares y subvenciones estatales y de la Diputación Provincial de Alicante, aparte de tener concierto con la seguridad social.

En el cuadro I se sintetiza la población de hecho (según el Censo de 1991) asistida en cada Distrito Sanitario. En general, ningún área alcanza la cifra de referencia establecida por el SERVASA como umbral adecuado de asistencia — entre doscientos mil y doscientos cincuenta mil habitantes-, aunque cabe advertir que las cifras demográficas están referidas al día 31 de marzo de 1991, es decir, en temporada baja para los espacios con fuerte afluencia de turistas en verano. No cabe duda que el contingente poblacional - por tanto, la demanda asistencial teórica a cubrir - se incrementa sustancialmente durante los meses estivales, sobre todo en los Distritos Sanitarios que se corresponden con el frente litoral alicantino. Por otro lado, en temporada turística baja existe también un importante contingente demográfico que habitualmente reside en la provincia pero que no se censa; y 
FIGURA 2. Isocronas a los Hospitales Generales (H) del SERVASA en la provincia de Alicante, por Distritos Sanitarios, en el estado actual de las carreteras (octubre 1992 - mayo 1993): A) Isocrona y tiempo (en minutos); B) Límite de Distrito Sanitario. En punteado, área integrada por municipios con menos de 2.000 habitantes en 1991. Fuente: Elaboración propia a partir de información proporcionada por fuentes municipales.

tampoco debe olvidarse el incremento de la demanda en invierno, sobre todo debido a las campañas vacacionales del INSERSO dirigidas a la Tercera Edad. Estos aspectos tienen especial incidencia igualmente en el frente costero (sobre todo en el área que cubre el Hospital de Villajoyosa).

Se han desglosado las cifras demográficas por umbrales isocrónicos respecto a cada centro hospitalario, de cinco en cinco minutos (más un último umbral homogéneo para los 
Cuadro I

POBLACIÓN ASISTIDA POR LOS HOSPITALES GENERALES DEL SERVASA EN LA PROVINCIA DE ALICANTE EN 1991, POR UMBRALES ISOCRÓNICOS

POBLACIÓN DE HECHO ASISTIDA

\begin{tabular}{|c|c|c|c|c|c|c|c|c|}
\hline \multirow[b]{3}{*}{ HOSPITAL } & \multicolumn{8}{|c|}{ POBLACIÓN DE HECHO ASISTIDA } \\
\hline & \multicolumn{8}{|c|}{ Tiempo de desplazamiento desde el lugar de residencia } \\
\hline & TOTAL & $<\mathbf{5}^{\prime}$ & $5-10^{\prime}$ & $10-15^{\prime}$ & $15-20^{\prime}$ & 20-25' & $25-30^{\prime}$ & $>\mathbf{3 0}$ \\
\hline Orihuela & 180.460 & 5.916 & 21.900 & 47.635 & 25.480 & 21.109 & 15.272 & 43.148 \\
\hline Elda & 191.605 & 108.573 & 16.383 & 46.708 & 3.052 & 8.807 & 5.759 & 2.323 \\
\hline Elche & 226.135 & 167.463 & 30.262 & 26.269 & 2.141 & 0 & 0 & 0 \\
\hline Alicante * & 173.076 & 137.800 & 26.915 & 2.779 & 5.276 & 0 & 0 & 64 \\
\hline San Juan * & 182.321 & 14.598 & 145.632 & 11.094 & 968 & 7.898 & 384 & 1.747 \\
\hline Alcoy & 131.748 & 63.869 & 11.277 & 8.392 & 2.207 & 22.622 & 7.893 & 15.488 \\
\hline Denia $* *$ & 110.429 & 25.023 & 21.513 & 13.413 & 6.054 & 19.781 & 10.262 & 14.383 \\
\hline Villajoyosa & 138.770 & 20.096 & 78.386 & 21.323 & 6.106 & 2.620 & 8.069 & 2.170 \\
\hline
\end{tabular}

NOTA GENERAL: Los tiempos empleados en los desplazamientos están calculados según trabajo de campo directo y en el estado actual de las carreteras (años 1992-93).

* Las cifras relativas a los Hospitales de Alicante y de San Juan están referidas a la situación sanitario administrativa y demográfica del año 1991. No se considera, por tanto, la nueva reordenación, efectuada en enero de 1994, según la cual el centro hospitalario de San Juan atenderá a 144.731 habitantes y el Hospital General de Alicante a 204.564 habitantes (véase Información, 21 de enero de 1994).

** Para el Hospital de Denia, sólo se considera la población residente en la provincia de Alicante.

FUENTE: Elaboración propia a partir de: I.N.E. DELEGACIÓN PROVINCIAL EN ALICANTE: Provincia de Alicante. Nomenclátor de viviendas, alojamientos, hogares y habitantes. Año 1991, inédito; e información proporcionada por fuentes municipales.

desplazamientos que requieren una inversión de tiempo superior a los treinta minutos) - véase cuadro I y fig. 2 - . Dada la fuerte concentración de la población alicantina en las cabeceras municipales, la ubicación de las mismas ha facilitado el diseño de las isocronas, mientras que para el espacio diseminado - muy importante en cifras absolutas y sobre todo relativas en determinados municipios, como Orihuela, Elche o Denia - ha sido de inestimable ayuda la consulta del Nomenclátor inédito del año 1991 confeccionado por la oficina de la Delegación Provincial del I.N.E. en Alicante ${ }^{22}$.

En general, se aprecia rapidez en la accesibilidad a todos los Hospitales, siendo los casos extremos Elche y Alicante, que tienen la práctica totalidad de la población asistida por debajo de la isocrona de 20 minutos. Los hospitales de Elda, San Juan y Villajoyosa responden al mismo esquema, caracterizándose igualmente por la rapidez del acceso, con al menos el $90 \%$ de los potenciales pacientes a menos de 20 minutos de distancia.

En el Hospital de Alcoy, un tercio de la población asistida necesita emplear sin embargo tiempo superior a los 20 minutos, dado que también cubre la demanda de poblaciones con estimable peso demográfico relativamente apartadas, en la Foia de Castalla (Onil, Castalla, Ibi).

22 Agradecemos al personal de la Oficina de la Delegación Provincial del I.N.E. en Alicante las facilidades brindadas para la consulta de dicha fuente. 
Por último, la población asistida por los hospitales de Denia y de San Bartolomé (Orihuela) se reparten aproximadamente por mitad a cada lado del umbral de los 20 minutos. En el caso de Denia es debido a la posición excéntrica del hospital dentro del Distrito Sanitario que jerarquiza y al mal estado general de las carreteras (sobre todo en la zona interior de la Marina Alta); en el caso del Bajo Segura, ello responde a la presencia de importantes núcleos de población al sur y en el frente costero de la comarca (San Miguel de Salinas, Pilar de la Horadada, Torrevieja, Guardamar), con el factor añadido del fuerte incremento estacional de residentes en verano; la puesta en funcionamiento a medio plazo de la nueva autovía Crevillente-Cartagena, atravesando de norte a sur las tierras más meridionales de la provincia de Alicante y con un ramal al centro hospitalario de San Bartolomé, contribuirá sin duda a mejorar la atención en estos espacios más alejados bajo el criterio de la accesibilidad al hospital.

Los espacios que se encuentran a mayor distancia de los Hospitales se corresponden — prácticamente en su totalidad - con las áreas más ruralizadas, más envejecidas y peor comunicadas de los respectivos ámbitos de cobertura asistencial. Ello ocurre sobre todo en la Marina Alta y en la Marina Baixa, en el interior del'Alcoià-Comtat y de l'Alacantí; también para el caso de los municipios con menos de 2.000 habitantes de la Vall de Beneixama, en el Alto Vinalopó, y del suroeste del Vinalopó Medio con respecto al Hospital de Elda, y con Tibi, en la Foia de Castalla, respecto al de San Juan. El escaso contingente poblacional que suman las localidades y áreas rurales actúa en contra de una mayor centralización geográfica de la infraestructura hospitalaria. El objetivo de acercar al máximo la asistencia médica especializada a los habitantes de estas zonas rurales más apartadas se subsana en buena medida con la existencia de Centros de Salud en Pego, Orba (previsto), Pedreguer, Gata, Callosa d'En Sarrià, Ibi y Biar.

Por otro lado, la población atendida por los hospitales alicantinos es, en su gran mayoría, una población urbana, muy concentrada en las cabeceras municipales, generalmente con fácil accesibilidad a los correspondientes centros hospitalarios.

La población que reside en los municipios «rurales» (todos los que censan menos de 2.000 habitantes) y en el diseminado o en núcleos secundarios pertenecientes a municipios con mayor rango demográfico, ofrecen cifras absolutas y relativas muy bajas dentro de los contextos geográfico-sanitarios en que se integran:

— En el Distrito que centraliza el Hospital de Elda son 9.773 habitantes los que residen en los municipios con menos de 2.000 hab. (5'1\% de la población total del Distrito) y 7.275 hab. los que residen en núcleos secundarios y diseminados (3'8\% del total del Distrito).

—Para el caso del Hospital de San Juan son 3.099 (1’7\%) y 7.660 hab (4’2\%) respectivamente.

- Para el de Alcoy son 8.977 (6’8\%) y 2.851 (2’2\%).

Cifras absolutas más elevadas pero porcentajes igualmente bajos ofrecen los efectivos en el medio rural de los Hospitales de:

- Villajoyosa, con 7.179 habitantes en municipios con menos de 2.000 hab (5'2\% del total del Distrito) y 18.046 hab en diseminado y núcleos secundarios (13’0\%).

- Alicante, con 12.333 hab en diseminado o en núcleos secundarios (7’1\%), no atendiendo a ningún municipio «rural».

— Elche, con 25.237 hab en su medio rural (11'2\%), ninguno en municipios con menos de 2.000 hab.

- Nuevamente, los centros hospitalarios de Denia y de San Bartolomé constituyen un modelo diferente al resto de los de la provincia de Alicante, sobre todo por el importante volumen de población residente en el ámbito rural: 
- Para el caso del Bajo Segura, 43.810 habitantes dispersos (24'3\% del total del Distrito) y $9.737(5,4 \%)$ en municipios «rurales».

-Dentro del ámbito de cobertura del Hospital de Denia se censan 23.678 efectivos dispersos (21'4\%) y 15.917 (14'4\%), respectivamente; estos últimos en su mayor parte residen en el área más alejada y peor comunicada de dicho Distrito, como ya quedó dicho.

\section{Estructura por edades de la población asistida por los centros hospitalarios de la provincia de Alicante}

Cuestión de máximo interés es la estructura por edades de las poblaciones que han de ser atendidas por los distintos hospitales. En el cuadro II se esquematiza el volumen y los porcentajes de los potenciales pacientes que, a priori, demandan mayor asistencia médica: la población más joven (entre 0 y 20 años de edad) y la más vieja (con 60 o más años).

Los cálculos por Distritos consideran: 1) las estructuras para el conjunto de efectivos humanos de cada unidad sanitaria; 2) para la población residente en territorios con menor accesibilidad a los respectivos centros hospitalarios, es decir, la ubicada por encima de la isocrona de 20 minutos, independientemente del rango demográfico; y 3) se distingue la estructura del total de población residente en municipios «rurales», con independencia de su ubicación geográfica.

Los cálculos efectuados, sobre población de derecho en 1991, permiten avanzar las siguientes conclusiones:

a) Entre la población más alejada de las instalaciones hospitalarias existe mayor grado

Cuadro II

POBLACIÓN JOVEN (0-20 AÑOS DE EDAD) Y VIEJA (CON 60 O MÁS AÑOS) ATENDIDA POR LOS HOSPITALES GENERALES DEL SERVASA EN LA PROVINCIA DE ALICANTE, POR DISTRITOS SANITARIOS (AÑO 1991)

\begin{tabular}{|c|c|c|c|c|c|c|c|c|c|c|c|c|}
\hline \multirow[b]{3}{*}{ HOSPITAL } & \multicolumn{6}{|c|}{ Población con menos de 20 años } & \multicolumn{6}{|c|}{ Población con 600 más años } \\
\hline & \multicolumn{2}{|c|}{1} & \multicolumn{2}{|c|}{2} & \multicolumn{2}{|c|}{3} & \multicolumn{2}{|c|}{1} & \multicolumn{2}{|c|}{2} & \multicolumn{2}{|c|}{3} \\
\hline & $\mathrm{N}^{0}$ & $\%$ & $\mathrm{~N}^{0}$ & $\%$ & $\mathrm{~N}^{0}$ & $\%$ & $\mathrm{~N}^{0}$ & $\%$ & $\mathrm{~N}^{0}$ & $\%$ & $\mathbf{N}^{0}$ & $\%$ \\
\hline Orihuela & 54.528 & 30,3 & 20.072 & 28,4 & 2.789 & 28,0 & 33.085 & 18,4 & 14.349 & 20,3 & 1.986 & 19,9 \\
\hline Elda & 59.625 & 31,0 & 4.594 & 27,6 & 2.452 & 25,2 & 34.316 & 17,8 & 3.984 & 23,9 & 2.614 & 26,9 \\
\hline Elche & 75.662 & 33,5 & - & - & - & - & 32.821 & 14,5 & - & - & - & - \\
\hline Alicante & 49.252 & 29,7 & - & - & - & - & 28.921 & 17,4 & - & - & - & - \\
\hline San Juan & 52.246 & 29,3 & 2.572 & 25,6 & 768 & 24,6 & 32.468 & 18,2 & 2.442 & 24,3 & 814 & 26,1 \\
\hline Alcoy & 37.040 & 27,7 & 13.706 & 29,4 & 1.761 & 18,9 & 26.831 & 20,1 & 8.362 & 18,0 & 3.052 & 32,8 \\
\hline Denia (a) & 27.483 & 25,0 & 10.182 & 23,2 & 3.101 & 19,3 & 25.968 & 23,7 & 11.800 & 26,9 & 5.299 & 33,0 \\
\hline Villajoyosa & 29.947 & 28,1 & 3.133 & 24,2 & 1.372 & 18,9 & 20.518 & 19,2 & 3.329 & 25,7 & 2.382 & 32,7 \\
\hline
\end{tabular}

1.- Todo el Distrito Sanitario.

2.- Conjunto de habitantes que residen por encima de la isocrona de $20^{\prime}$.

3.- Conjunto de municipios con menos de 2.000 hab.; el porcentaje está referido al conjunto de la respectiva población rural.

NOTA GENERAL: Los tiempos empleados en los desplazamientos están calculados según el estado actual de las carreteras (años 1992-93).

(a) Para Denia, sólo se considera la población residente en la provincia de Alicante.

FUENTE: Elaboración propia a partir de: I.N.E. DELEGACIÓN PROVINCIAL EN ALICANTE: Provincia de Alicante. Nomenclátor de viviendas, alojamientos, hogares y habitantes. Año 1991, inédito; e información proporcionada por fuentes municipales. 
Figura 3. Porcentajes de población con 60 o más años de edad sobre el total de población de derecho en los municipios con menos de 2.000 habitantes (Censo de Población de 1991).

de envejecimiento en casi todos los Distritos considerados, pues sus porcentajes de población joven son más bajos que el promedio global de cada territorio, mientras los porcentajes de población con 60 ó más años son superiores a las respectivas medias.

Sólo el caso de Alcoy escapa a la regla, lo que se debe a la incidencia que sobre el conjunto de la población residente a más de 20 minutos del hospital alcoyano tienen municipios con importantes volúmenes demográficos en la Foia de Castalla. Así, cabe distinguir entre los porcentajes de Ibi, con 20.685 habitantes de derecho — de ellos 6.441 con menos de 20 años de edad (31'1\%) y 3.121 con 60 ó más años (15'1\%)—, Castalla, con 7.229 hab -2.188 con menos de 20 años (30'3\%) y 1.238 en edad senil (17'1\%) —, y Onil, con 6.631 hab y porcentajes de población joven -2.099 hab- del 31 '7\% y de población senil -1.061 hab- del 16’0\%. 
En contraposición, las cifras de población del conjunto de los doce municipios del interior de l'Alcoià-Comtat que distan más de 20 minutos del hospital de la ciudad de Alcoy son las que siguen: 5.010 hab de derecho en total, con una media de 418 por municipio, siendo los promedios del 18'5\% para el contingente con menos de 20 años (926 individuos) y 33’3\% para el grupo más envejecido (1.066 hab con 60 o más años de edad en conjunto).

b) El envejecimiento es especialmente acusado entre los municipios «rurales» de menor censo, en cualquiera de los ámbitos geográfico-sanitarios considerados (mapa 3). Existen mayores diferencias entre las medias relativas de estas localidades - la mayor parte por debajo de los 1.000 hab- y los respectivos promedios territoriales, que las apreciadas en el párrafo anterior con referencia al umbral isocrónico por encima de los 20 minutos. Se trata de desviaciones porcentuales — en algunos casos muy fuertes - a la baja entre la población joven y muy superiores a las medias comarcales entre la población senil.

Cuadro III

POBLACIÓN DE DERECHO CON 60 O MÁS AÑOS DE EDAD EN 21 MUNICIPIOS CON MENOS DE 2.000 HAB.EN 1991 (AÑOS1960 Y 1991)

\begin{tabular}{|c|c|c|c|c|c|c|}
\hline \multirow[b]{2}{*}{ Municipios } & Pobl. & & & Pobl. & $\begin{array}{r}1991 \\
\text { Pob } \\
>=\end{array}$ & \\
\hline & Total (a) & $\mathbf{N}^{\circ}$ & $\%$ & Total & $\mathbf{N}^{\mathbf{o}}$ & $\%$ \\
\hline Algueña & 1.735 & 317 & 18,3 & 1.490 & 387 & 26,0 \\
\hline H. Nieves & 1.805 & 363 & 20,1 & 1.426 & 396 & 27,8 \\
\hline La Romana & 2.100 & 418 & 19,9 & 1.909 & 527 & 27,6 \\
\hline Benejama & 2.289 & 473 & 20,7 & 1.819 & 525 & 28,9 \\
\hline Daya Nva. (b) & 1.258 & 111 & 8,8 & 1.199 & 238 & 19,8 \\
\hline Gr.Rocamora & 1.846 & 173 & 9,4 & 1.961 & 366 & 18,7 \\
\hline Algorfa & 1.019 & 78 & 7,7 & 1.093 & 192 & 17,6 \\
\hline Jacarilla & 1.162 & 146 & 12,6 & 1.466 & 291 & 19,8 \\
\hline Torremanzanas & 1.090 & 257 & 23,6 & 736 & 240 & 32,6 \\
\hline Tibi & 1.159 & 174 & 15,0 & 1.045 & 240 & 23,0 \\
\hline V. Ebo & 584 & 111 & 19,0 & 359 & 161 & 44,8 \\
\hline Benitachell & 1.614 & 323 & 20,0 & 1.641 & 469 & 28,6 \\
\hline R.Almunia & 506 & 113 & 22,3 & 376 & 150 & 39,9 \\
\hline Orba & 1.255 & 222 & 17,7 & 1.499 & 498 & 33,2 \\
\hline Benimantell & 617 & 97 & 15,7 & 453 & 144 & 31,8 \\
\hline Benifato & 200 & 53 & 26,5 & 124 & 44 & 35,5 \\
\hline Gayanes & 499 & 97 & 19,4 & 331 & 113 & 34,1 \\
\hline Beniarrés & 1.857 & 313 & 16,9 & 1.503 & 401 & 26,7 \\
\hline Benimassot & 229 & 56 & 24,5 & 118 & 45 & 38,1 \\
\hline Balones & 301 & 56 & 18,6 & 152 & 61 & 40,1 \\
\hline Alcocer Pl. & 223 & 48 & 21,5 & 123 & 48 & 39,0 \\
\hline
\end{tabular}

(a) Población con edad conocida.

(b) En 1960, sin datos de Puebla de Rocamora.

FUENTE: Elaboración propia a partir del Padrón Municipal de Población del año 1960, y de INSTITUT VALENCIA D'ESTADISTICA. Resultados a nivel comarcal y provincial de los censos de población y viviendas 1991 en la Comunidad Valenciana, Valencia, 1992. 
El envejecimiento de la población rural se ha disparado en cifras relativas durante los últimos decenios, como demuestra el cuadro III, donde se recogen cifras absolutas y porcentajes de población con 60 o más años de edad en una muestra aleatoria de 21 municipios con menos de 2.000 hab. en 1991, dsitribuidos por toda la provincia de Alicante. Se demuestra claramente cómo se han incrementado dichos porcentajes (en algunos casos, también las cifras absolutas) en la totalidad de casos considerados, entre 1960 y 1991.

Este envejecimiento, consecuencia de décadas sucesivas de éxodo rural, es un tema prioritario a la hora de planificar la política asistencial en estas comunidades rurales con menor censo de la provincia de Alicante.

Como bien afirma G. Fernández-Mayoralas, una de las consecuencias del envejecimiento en un espacio dado es la inadaptación de los equipamientos y servicios destinados a los distintos grupos de edades. «Los servicios sanitarios, en concreto, ofrecen un interés relevante por cuanto la salud o el bienestar de la población anciana contiene unas connotaciones especiales relacionadas con la edad, que se vinculan directamente con la cuestión de la accesibilidad espacial y temporal. Es justamente la población con menores posibilidades para moverse y, por tanto, no sólo la localización de los servicios médicos, sino los de asistencia social en general, debe ser objeto de una planificación que persiga su mayor acercamiento a este contingente de población más envejecida» ${ }^{23}$.

Merece la pena, por tanto, recordar la infraestructura médica existente en la actualidad (1993) en los municipios alicantinos con menos de 2.000 habitantes.

\section{Infraestructura y medios para la atención a la salud en municipios «rurales» de la provincia de Alicante}

En estos municipios la infraestructura suele reducirse a la presencia de un consultorio de asistencia primaria (fig. 4). En cuarenta municipios con este rango demográfico este tipo de instalación depende directamente del SERVASA. En el resto (40 municipios), se utilizan locales habilitados para las consultas - no siempre diarias-, propiedad de los respectivos Ayuntamientos o reconvertidos para este uso, mediante convenios con dicho organismo público, a partir de otra clase de infraestructura (antiguas viviendas de maestros, aulas escolares, etc.). Sólo tres localidades con menos de 2.000 hab cuentan con Consultorio de Verano - Alquería de Aznar, San Fulgencio y Finestrat-, mientras que cinco poblaciones «rurales» de la Marina Baixa y del Bajo Segura se integran dentro del Programa de Extracción Periférica de productos para la realización de Análisis Clínicos ${ }^{24}$.

Por otra parte, la presencia o no de personal médico censado en municipios alicantinos con menos de 2.000 hab, no guarda una estrecha relación con el censo de cada localidad (es decir, con el número de potenciales pacientes). Existen, por un lado, cuarenta y un municipios «rurales» que cuentan con médico censado en la propia localidad; en general, en este caso se incluyen todos los de más de 1.000 hab, si bien también disponen de personal colegiado otras con contingente demográfico muy reducido (menos de 250 hab.).

23 FERNÁNDEZ-MAYORALAS FERNÁNDEZ, G.: Op. cit., p. 185.

24 El Programa de Extracciones Periféricas de productos para la realización de Análisis Clínicos (diseñado por la Conselleria de Sanitat i Consum de la Generalitat Valenciana) pretende que, en las localidades que carecen de laboratorios propios para realizar las determinaciones analíticas, la población no tenga que efectuar desplazamientos hasta el centro hospitalario más próximo, sino a un determinado número de localidades, repartidas por toda la Comunidad Valenciana, en cuyos Centros de Salud se podrá llevar a cabo la obtención de las muestras biológicas necesarias y su envío al laboratorio que ha de analizarlas. 
Figura 4. Centros Hospitalarios en la provincia de Alicante (A). Insfraestructura sanitaria de carácter público (SERVASA) en municipios con menos de 2.000 habitantes en 1991: (B) Consultorio de Asistencia Primaria; (C) Consultorio de Verano; (D) Localidad incluida en el Programa de Extracción Periférica de productos para la realización de Análisis Clínicos. Fuente: CONSELLERIA DE SANITAT I CONSUM: Guía de servicios de Salud, Valencia, 1989, pp. 151-221, y actualización a partir de información proporcionada por fuentes municipales.

Dado que en la mayor parte de los casos sólo se registra un médico por municipio, la correlación, en 1988, entre número de habitantes y colegiados (mapa 5) es muy dispar, oscilando entre más de 1.500 (La Romana, Beniarrés, San Fulgencio) y menos de 250 en algunos municipios de la Montaña (Agres — con dos médicos censados-, Penáguila, Balones, Alcolecha) y ambas Marinas (Vall de Gallinera - que censa tres facultativos-, Vall de Ebo, Benimantell). Algunos de estos profesionales también deben atender a la población de localidades vecinas carentes de médico propio. 
FiguRA 5. Número de habitantes por médico residente en municipios con menos de 2.000 habitantes en la provincia de Alicante (1989): 1) Municipios sin personal médico censado (la flecha indica la localidad de procedencia del médico); 2) Menos de 250; 3) 250-499; 4) 500-999; 5) 1.000-1.499; 6) 1.500 o más. Fuente: C.A.M.: Datos y series estadísticas, 1988-1989, e información proporcionada por fuentes municipales.

Efectivamente, existe una relación de treinta y nueve municipios «rurales» donde la asistencia corresponde a colegiados procedentes de otros lugares, tal y como se cartografía en el gráfico 5. Coincide el listado de estos últimos generalmente con las localidades situadas en la zona más interior y montañosa de la provincia, con una fuerte dispersión demográfica en pequeños núcleos rurales y una red de carreteras lenta. Suele coincidir, por otra parte, como ya se ha referido, con el hecho de tratarse de localidades que, dada la ubicación geográfica de los hospitales de Alcoy, Denia, Villajoyosa y San Juan, se inclu- 
yen en los umbrales isocrónicos superiores a los 20 minutos —incluso más de 30 minutos- para desplazarse a los respectos centros hospitalarios comarcales.

No hay que olvidar, además, los elevados porcentajes de vejez que suelen producirse en los mismos municipios: más del $30 \%$ de la población que reside en prácticamente todos ellos tiene sesenta o más años de edad, y en algunos casos más del 40 ó el 50 por ciento (véase mapa 3).

$$
* * * * * * *
$$

Las infraestructuras, dotaciones y servicios que existen en un espacio dado constituyen un baremo de gran interés por cuanto su presencia o no, así como la calidad del servicio, puede actuar como factor impulsor o retardador de todo el proceso económico de un municipio. Es norma común que los espacios más ruralizados —entre los que se debe incluir a los municipios con menos de 2.000 habitantes - parten con desventaja comparativa en lo que respecta a la satisfacción de los servicios básicos de la población. Así, en el plano sanitario, no sólo debe considerarse el hecho de contar casi de manera exclusiva con la infraestructura asistencial más elemental y con el censo más pobre de personal sanitario, sino que también hay que tener presente su estructura de edad muy envejecida y su menor accesibilidad —en comparación con otros espacios menos ruralizados- a los centros sanitarios mejor dotados (los hospitales).

\section{Fuentes estadísticas y bibliografía fundamental}

CAJA DE AHORROS DEL MEDITERRÁNEO: Datos y Series Estadísticas, vol. 1988-89.

CONSELLERIA D'ECONOMIA I HISENDA: Comunitat Valenciana. Estadística Municipal, Valencia, 1988.

CONSELLERIA DE SANITAT I CONSUM: Guía de Servicios de Salud, Valencia, 1989.

CONSELLERIA DE SANITAT I CONSUM: Cartografía de los Recursos Sanitarios de la Comunidat Valenciana, Valencia, 1990.

CONSELLERIA DE SANITAT I CONSUM: Libro Blanco de la Sanidad de la Comunidad Valenciana, Valencia, 1991.

GENERALITAT VALENCIANA: Ley del Servicio Valenciano de Salud, Servicio de Publicaciones, Valencia, 1988.

INSTITUTO NACIONAL DE ESTADÍSTICA. DELEGACIÓN PROVINCIAL EN ALICANTE: Provincia de Alicante. Nomenclátor de viviendas, alojamientos, hogares y habitantes. Año 1991, inédito.

INSTITUT VALENCIA D’ESTADISTICA: Comunitat Valenciana. Estadística Municipal, vol. 4 (Valencia, 1990) y 5 (Valencia, 1991).

INSTITUT VALENCIA D'ESTADISTICA. Resultados a nivel comarcal y provincial de los censos de población y viviendas 1991 en la Comunidad Valenciana, Valencia, 1992.

PÉREZ, S. et al:: Poblaciones por Áreas de Salud de la Comunidad Valenciana, Servicio de Publicaciones de la Generalitat Valenciana, Valencia, 1988.

PREVASA: Situación actual, problemas y perspectivas de las comarcas valencianas, vols. XIX (La Marina Alta), XX (L'Alcoià), XXIa (L'Alt Vinalopó), XXIb (El Baix Vinalopó), XXII (La Marina Baixa), XXIII (L'Alacantí) y XXIV (La Vega Baja del Segura), Valencia, 1982.

SANZ CAÑADA, E. (dir.): El equipamiento social, en PREVASA: Estudios básicos para la ordenación del territorio de la Comunidad Valenciana, vol. IV, Valencia, 1982.

SERVASA: Indicadores. Centros de Salud. Informe Anual, Valencia, 1989.

SERVASA: Indicadores de Gestión. Asistencia Especializada, 2 vols., Valencia, 1989. 